EFI $95-47$

$\mathrm{MPI}-\mathrm{Ph} / 95-81$

\title{
Reduction of Coupling Parameters [
}

\author{
Reinhard Oehme \\ Enrico Fermi Institute and Department of Physics \\ University of Chicago, Chicago, Illinois, 60637, USA 9 \\ and \\ Max-Planck-Institut für Physik \\ - Werner-Heisenberg-Institut - \\ 80805 Munich, Germany
}

\begin{abstract}
The general theory of the reduction in the number of coupling parameters is discussed. The method involves renormalization group invariant relations between couplings. It is more general than the imposition of symmetries. There are reduced theories with no known symmetry. The reduction scheme is finding many applications. Discussed in some detail are the construction of gauge theories with "minimal" coupling for Yang-Mills and matter fields, and the Gauge-Yukawa Unification within N=1 supersymmetric GUT's.
\end{abstract}

\footnotetext{
${ }^{1}$ Plenary talk presented at the XVIIIth International Workshop on High Energy Physics and Field Theory, Moscow-Protvino, June 1995. To be published in the Proceedings.

${ }^{2}$ Permanent Address
} 


\section{INTRODUCTION}

In recent years, the method of the reduction in the number of coupling parameters of renormalizable field theories [1, 2] has found many applications, theoretical and phenomenological. In this talk, we will briefly review [3] the reduction scheme, and report about some of the applications.

Lagrangians of quantum field theories can be constrained by renormalizability requirements. Generally, this leaves a large number of independent coupling parameters. We consider the possibility to reduce this number by imposing relations between the dimensionless couplings such that renormalizability is preserved, and that the relations are independent of the normalization point. We require that the original, as well as the reduced theories satisfy the corresponding renormalization group equations. The resulting set of differential equations, the reduction equations, are necessary and sufficient for the renormalization group invariance of the reduced theory.

A standard method for the reduction in the number of couplings is the imposition of symmetries. The reduction method described above is more general. It includes possible solutions reflecting symmetries, but also provides other reductions which have no known connections with any symmetry.

In the following, we list some of the applications of the reduction method:

* Construction of gauge theories with "minimal" coupling of Yang-Mills and matter fields [团.

* Classification of renormalizable theories with a single coupling parameter [1].

* Proof of conformal invariance (finiteness) for $N=1$ SUSY gauge theories with vanishing lowest order $\beta$-function on the basis of one-loop information [5]. 
* Reduction of the infinite number of coupling parameters appearing in the light-cone quantization method [6].

* Gauge-Yukawa unifications within the framework of SUSY GUT's. Successful calculations of top-quark and bottom-quark masses within this framework [7].

* Applications of reduction to the standard model (non-SUSY) give values for the top-quark mass which are too small, indicating the need for more matter fields [8, 9].

* Universal soft breaking of supersymmetry. Renormalization group invariant relations between soft SUSY-breaking parameters [10].

There are many other applications. The reduction of Yukawa couplings may also be of interest in connection with duality and IR-fixed points in $N=1$ SUSY gauge theories.

\section{REDUCTION SCHEME}

Let us consider a renormalizable theory with $n+1$ real, independent dimensionless parameter, $\lambda, \lambda_{1}, \ldots, \lambda_{n}$. We call $\lambda$ the "primary coupling", and consider the reduction to one coupling parameter by the requirement

$$
\lambda_{k}=\lambda_{k}(\lambda), \quad k=1, \ldots, n
$$

The Green's functions of the reduced theory should then satisfy the renormalization group equation

$$
\left(\kappa^{2} \frac{\partial}{\partial \kappa^{2}}+\beta_{\lambda}(\lambda) \frac{\partial}{\partial \lambda}+\gamma_{G}(\lambda)\right) G\left(k_{i}, \kappa_{2}, \lambda\right)=0,
$$

where $G$ is defined in terms of the multi-parameter Green's functions by

$$
G\left(k_{i}, \kappa^{2}, \lambda\right)=G\left(k_{i}, \kappa^{2}, \lambda, \lambda_{1}(\lambda), \ldots, \lambda_{n}(\lambda)\right)
$$


with corresponding expressions for the coefficients:

$$
\begin{aligned}
\beta_{\lambda}(\lambda) & =\beta_{\lambda}\left(\lambda, \lambda_{1}(\lambda), \ldots, \lambda_{n}(\lambda)\right) \\
\gamma_{G}(\lambda) & =\gamma_{G}\left(\lambda, \lambda_{1}(\lambda), \ldots, \lambda_{n}(\lambda)\right) .
\end{aligned}
$$

The Green's functions of the original theory satisfy the equations

$$
\left(\kappa^{2} \frac{\partial}{\partial \kappa^{2}}+\beta_{\lambda} \frac{\partial}{\partial \lambda}+\sum_{k=1}^{n} \beta_{k} \frac{\partial}{\partial \lambda_{k}}+\gamma_{G}\right) G=0,
$$

where $G=G\left(k_{i}, \kappa^{2}, \lambda, \lambda_{1}, \ldots, \lambda_{n}\right), \beta_{\lambda}=\beta_{\lambda}\left(\lambda, \lambda_{1}, \ldots, \lambda_{n}\right)$, etc. . By substitution, we find then the reduction equations:

$$
\beta_{\lambda}(\lambda) \frac{d \lambda_{k}(\lambda)}{d \lambda}=\beta_{k}(\lambda), \quad k=1, \ldots, n
$$

as necessary and sufficient conditions for the validity of the renormalization group equations (2) for the reduced theory.

We consider here massless theories, or theories with mass independent renormalization schemes, so that no mass parameters occur in the coefficient functions of the renormalizaton group equation. The perturbative renormalizability of the multi-parameter theory implies then that the $\beta$-functions have asymptotic power series expansions at $\lambda=\lambda_{k}=0$. For the models considered, these expansions can be written the form

$$
\beta_{\lambda}\left(\lambda, \lambda_{1}, \ldots, \lambda_{n}\right)=\beta_{\lambda 0} \lambda^{2}+\left(\beta_{\lambda 1} \lambda^{3}+\beta_{\lambda 1, k} \lambda_{k} \lambda^{2}+\beta_{\lambda 1, k k^{\prime}} \lambda_{k} \lambda_{k^{\prime}} \lambda\right)+\cdots
$$

and

$$
\beta_{k}\left(\lambda, \lambda_{1}, \ldots, \lambda_{n}\right)=\left(c_{k}^{(0)} \lambda^{2}+c_{k, k^{\prime}}^{(0)} \lambda_{k^{\prime}} \lambda+c_{k, k^{\prime} k^{\prime \prime}}^{(0)} \lambda_{k^{\prime}} \lambda_{k^{\prime \prime}}\right)+\cdots
$$

We note that $\beta_{\lambda}$ vanishes for $\lambda \rightarrow 0$.

Since $\beta_{\lambda}(\lambda)$ generally will have a zero at $\lambda=0$, this is a singular point of the reduction equations and the usual existence theorems are not applicable 
here. Without further constraints, we may have general solutions with as many free parameters as there are couplings. These are not of direct use for the reduction, but will be considered later in connection with small coupling treated as corrections. For the actual reduction, we make the assumption that the Green's functions of the reduced theory have power series expansion in $\lambda$. To this end, we require that the couplings $\lambda_{k}(\lambda)$ are given by asymptotic power series for $\lambda \rightarrow 0$.

\section{SPECIAL SOLUTIONS}

With all couplings $\lambda, \lambda_{k}$ vanishing on the weak coupling limit of the original theory, we assume that

$$
\lambda_{k}(\lambda)=\lambda f_{k}(\lambda), \quad k=1, \ldots, n,
$$

with bounded functions $f_{k}(\lambda)$ in some interval $0 \leq \lambda \leq \lambda_{0}$. It is then useful to write the reduction equations (6) in terms of $f_{k}(\lambda)$ :

$$
\beta_{\lambda}\left(\lambda \frac{d f_{k}}{d \lambda}+f_{k}\right)=\beta_{k},
$$

with $f_{k}(\lambda)$ given by the power series expansions

$$
f_{k}(\lambda)=f_{k}^{0}+\sum_{m=1}^{\infty} \chi_{k}^{(m)} \lambda^{m} .
$$

It is convenient in the following to use the notation

$$
\begin{aligned}
& \beta_{\lambda}(\lambda)=\beta_{\lambda}\left(\lambda, \lambda f_{i}(\lambda)\right)=\sum_{n=0}^{\infty} \beta_{\lambda n}(f) \lambda^{n+1}, \\
& \beta_{k}(\lambda)=\beta_{k}\left(\lambda, \lambda f_{i}(\lambda)\right)=\sum_{n=0}^{\infty} \beta_{k}^{(n)}(f) \lambda^{n+2} .
\end{aligned}
$$


Now we substitute the expansion (11) into the reduction equations (10). In lowest order, using the notation of (12) and (13), we obtain the one-loop relations [1, 2] :

$$
\beta_{\lambda 0}\left(f^{0}\right) f_{k}^{0}-\beta_{k}^{(0)}\left(f^{0}\right)=0
$$

or, in explicit form, with the coefficients as defined in (8):

$$
c_{k}^{(0)}+\left(c_{k, k^{\prime}}^{(0)}-\beta_{\lambda 0} \delta_{k k^{\prime}}\right) f_{k^{\prime}}^{0}+c_{k, k^{\prime} k^{\prime \prime}}^{(0)} f_{k^{\prime}}^{0} f_{k^{\prime \prime}}^{0}=0
$$

Here, and in the following, appropriate summation over equal indices is understood.

The equations (14) are the fundamental relations for special reductions. One loop criteria also decide whether the higher order coefficients are determined by the reduction equations. Up to $m+1$ loops, we have the equations:

$$
\left(M_{k k^{\prime}}\left(f^{0}\right)-m \beta_{\lambda 0} \delta_{k k^{\prime}}\right) \chi_{k^{\prime}}^{(m)}=\left(\beta_{\lambda m}\left(f^{0}\right) f_{k}^{0}-\beta_{k}^{(m)}\left(f^{0}\right)\right)+X_{k}^{(m)},
$$

where $m=1,2, \ldots, \quad k=1, \ldots, n$. The matrix $M\left(f^{0}\right)$ is given by

$$
M_{k k^{\prime}}\left(f^{0}\right)=c_{k, k^{\prime}}^{(0)}+2 c_{k, k^{\prime} k^{\prime \prime}}^{(0)} f_{k^{\prime \prime}}^{0}-\delta_{k k^{\prime}} \beta_{\lambda 0} .
$$

The rest term $X^{(m)}$ depends only upon the coefficients $\chi^{(1)}, \ldots, \chi^{(m-1)}$, and upon the $\beta$-function coefficients in (12) and (13), evaluated at $f_{k}=f_{k}^{0}$, for order $m-1$ and lower. They vanish for $\chi^{(1)}=\ldots=\chi^{(m-1)}=0$.

We see that the one-loop criteria

$$
\operatorname{det}\left(M_{k k^{\prime}}\left(f^{0}\right)-m \beta_{\lambda 0} \delta_{k k^{\prime}}\right) \neq 0 \text { for } m=1,2, \ldots
$$

are sufficient to insure that all coefficients $\chi^{(m)}$ in the expansion (11) are determined. Then the reduced theory has a renormalized power series expansion in $\lambda$. All possible solutions of this kind are determined by the one-loop equation (14) for $f_{k}^{0}$. 
If the conditions (18) are satisfied, and all coefficients $\chi^{(m)}$ are determined, we can use regular reparametrization transformations in order to remove all higher terms in the expansion (11). The reparametrization transformations are of the form

$$
\begin{aligned}
& \lambda^{\prime}=\lambda^{\prime}\left(\lambda, \lambda_{1}, \ldots, \lambda_{n}\right)=\lambda+a^{(20)} \lambda^{2}+a_{k}^{(11)} \lambda_{k} \lambda+\cdots, \\
& \lambda_{k}^{\prime}=\lambda_{k}^{\prime}\left(\lambda, \lambda_{1}, \ldots, \lambda_{n}\right)=\lambda_{k}+b_{k k^{\prime} k^{\prime \prime}}^{(20)} \lambda_{k^{\prime}} \lambda_{k^{\prime \prime}}+b_{k k^{\prime}}^{(11)} \lambda_{k^{\prime}} \lambda+\cdots .
\end{aligned}
$$

They leave invariant the one-loop quantities

$$
f_{k}^{0}, \beta_{\lambda 0}\left(f^{0}\right), \beta_{k}^{(0)}\left(f^{0}\right), M_{k k^{\prime}}\left(f^{0}\right)
$$

but not the coefficients $\chi^{(m)}$, which can be transformed to zero if they are uniquely determined by (16). Then we have a frame in coupling parameter space, where the special solutions are of the form

$$
\lambda_{k}(\lambda)=\lambda f_{k}^{0}
$$

with the quantities $f_{k}^{0}$ being given exactly as solutions of the one-loop reduction equations (14). In many (but not all) cases, their exist only a few solutions $f_{k}^{0}$, and hence corresponding reduced theories with power series expansions. In particular, this is the case after the imposition of simple physical requirement, like positivity etc..

In the special cases where $f_{k}^{0}=0$, and $\chi_{k}^{(m)}=0$ for $m<N$, but $\chi_{k}^{(N)} \neq 0$, we find that

$$
f_{k}(\lambda)=\chi_{k}^{(N)} \lambda^{N}, \quad N \geq 1
$$

after appropriate reparametrization.

Let us now consider the situation where the matrix $\beta_{\lambda 0}^{-1} M_{k k^{\prime}}\left(f^{0}\right)$ has one or more positive integer eigenvalues, so that the determinant (18) vanishes. 
Suppose there is a positive eigenvalue for $m=N \geq 1$ and $\beta_{\lambda 0} \neq 0$. Then we find that the asymptotic power series must be supplemented by terms of the form $\lambda^{m}(\lg \lambda)^{p}$, with $m \geq N$ and $1<p<\sigma(N)$ [3], 9]. After reparametrization, we obtain then an expansion of the form

$$
f_{k}(\lambda)=f_{k}^{0}+\chi_{k}^{(N, 1)} \lambda^{N} \lg \lambda+\chi_{k}^{(N)} \lambda^{N}+\ldots,
$$

where all parameters are determined except the vector $\chi_{k}^{(N)}$, which contains $r$ free parameters if the eigenvalue has $r$-fold degeneracy. We have a well defined, "renormalized" theory with logarithmic terms in the asymptotic expansion for $\lambda \rightarrow 0$.

We will discuss application in a later section. Here we mention only the most simple example of special reduction, a model with Yukawa and quartic coupling:

$$
\mathcal{L}_{\text {int }}=i \sqrt{\lambda} \bar{\psi} \gamma_{5} \psi \phi-\frac{\lambda_{1}}{4 !} \phi^{4}
$$

With $\lambda$ as the primary coupling, the reduction results in the solutions $\lambda_{1 \pm}=$ $\lambda f_{1 \pm}^{0}$ with $f_{1 \pm}^{0}=\frac{1}{3}(1 \pm \sqrt{145})$. The matrix is $\beta_{\lambda 0}^{-1} M_{11}\left(f^{0}\right)= \pm \sqrt{145} / 5$, so that there is no positive eigenvector. Since $\lambda_{1}$ should be positive, the unique, relevant solution is $\lambda_{1}=\lambda \frac{1}{3}(1+\sqrt{145})$.

\section{GENERAL SOLUTIONS}

For systems with non-vanishing determinant (18) for all $m=1,2, \ldots$, we may have general solutions of the reduction equations which approach the special solutions $\lambda_{k}(\lambda)=\lambda f_{k}^{0}$ for $\lambda \rightarrow 0$. For simplicity, and as a charac-

teristic example, let us assume that $\beta_{\lambda 0} \neq 0$, and that $\beta_{\lambda 0}^{-1} M\left(f^{0}\right)$ has one positive, non-integer eigenvalue $\eta>0$, all other eigenvalues being negative. 
Then we find general solutions of the form

$$
f_{k}(\lambda)=f_{k}^{0}+\sum_{a, b} \chi_{k}^{(a \eta+b)} \lambda^{a \eta+b}+\sum_{m} \chi_{k}^{(m)} \lambda^{m}
$$

with $a=1,2, \ldots, b=0,1, \ldots, a \eta+b=$ non-integer. After reparametrization, powers with $m<\eta$ are removed, and we have $f_{k}(\lambda)=f_{k}^{0}+\chi_{k}^{(\eta)} \lambda^{\eta}+\ldots$ , which is of particular interest in situations where $f_{k}^{0}=0$, and where the corresponding couplings can be considered as small corrections. This will be discussed later.

In (25), all coefficients are determined except $\chi_{k}^{(\eta)}$, which contains $r$ arbitrary parameters if the eigenvalue has $r$-fold degeneracy. With

$$
\beta_{\lambda 0}^{-1} M_{k k^{\prime}}\left(f^{0}\right) \xi_{k^{\prime}}^{(i)}=\eta \xi_{k}^{(i)}, \quad i=1, \ldots, r
$$

we can write this coefficient in terms of the eigenvectors in the form

$$
\chi_{k}^{(\eta)}=C_{1} \xi_{k}^{(1)}+\ldots+C_{r} \xi_{k}^{(r)}
$$

where the undetermined parameters are exhibited. These considerations can be generalized to cases with several positive eigenvalues. In special situations, also $\operatorname{logarithmic}$ factors $(\lg \lambda)$, and powers thereof, may be required [9, 3].

For our previously discussed example with Yukawa and quartic coupling, we have a positive eigenvalue for the solution $\lambda_{1+}(\lambda)$, which is given by $\eta=\sqrt{145} / 5$. Consequently, there is a general solution $f_{1+}(\lambda)=f_{1+}^{0}+$ $C_{+} \lambda^{\sqrt{145} / 5}+\chi^{(3)} \lambda^{3}+\ldots$, with $C_{+}$arbitrary, and the other coefficients being determined. We see that here the power series solution $f_{1+}(\lambda)=f_{1+}^{0}$ is a stable solution. All general solutions also tend to $f_{1+}^{0}$ for $\lambda \rightarrow 0$,

In the following, we consider briefly a phenomenological application of general solutions. For some systems it is not possible to perform a complete 
reduction leaving only a single coupling constant, but it may be sensible to treat a subset of coupling parameters as small perturbations, while reducing all others. Suppose we consider the parameters $f_{a}(\lambda)=\lambda_{a}(\lambda) \lambda^{-1}, \quad a=$ $1, \ldots, n^{\prime}, n^{\prime}<n$ as small, and the rest of the couplings, $f_{\alpha}(\lambda)=\lambda_{\alpha}(\lambda) \lambda^{-1}$, $\alpha=n^{\prime}+1, \ldots, n$ as large couplings. If $f_{a}(\lambda) \equiv 0$, we have an undisturbed system involving only the large couplings $f_{\alpha}$, and we can look for special solutions as power series in $\lambda$, with $f_{\alpha}^{0}>0, \lambda_{\alpha}=\lambda f_{\alpha}^{0}$.

If we now want to include the small couplings $f_{a}(\lambda)$ as corrections, we look for solutions of the full set of reduction equations, assuming $f_{a}^{0}=0$ for the small couplings. Under these circumstances, it is reasonable to treat $f_{\alpha}$ as functions of $\lambda$ and as functionals of the small couplings $f_{a}(\lambda)$. Then we can rewrite the full set of reduction equations (10) as partial differential equations for $\left.f_{\alpha}\left(\lambda, f_{a}(\lambda)\right) \llbracket 7,8\right]$ :

$$
\beta_{\lambda} \lambda \frac{\partial f_{k}}{\partial \lambda}+\sum_{a=1}^{n^{\prime}} \frac{\partial f_{\alpha}}{\partial f_{a}}\left(\beta_{a}(f)-\beta_{\lambda} f_{a}\right)=\beta_{\alpha}(f)-\beta_{\lambda} f_{\alpha},
$$

with solutions $f_{\alpha}(\lambda)$, which are power series in $\lambda$ :

$$
f_{\alpha}(\lambda)=f_{\alpha}^{0}+\sum_{m=0} \lambda^{m} \chi_{\alpha}^{(m)}\left(f_{a}(\lambda)\right)
$$

The equations (28) are equivalent to the corresponding original reduction equations (10).

As a consequence of the fact that, for $f_{a}(\lambda) \equiv 0$, we have the usual special solution of the subsystem of large couplings, the coefficients $\chi^{(m)}\left(f_{a}\right)$ can be considered as power series in $f_{a}$ [11]. Inserting (29) into (28), we find that lowest order information is again sufficient to provide conditions for the uniqueness of the solutions. For simplicity, let us make the assumption, which is not unrealistic for applications, that the matrix $\beta_{\lambda 0}^{-1} M\left(f_{\alpha}^{0}\right)$ is diagonal, and 
that the eigenvalues $\eta^{(a)}=\beta_{\lambda 0}^{-1} M_{a a}\left(f_{\alpha}^{0}\right)$ are all positive. With $f_{a}^{0}=0$, the leading behavior of $f_{a}(\lambda)$ for $\lambda \rightarrow 0$ is then $f_{a}(\lambda) \cong C_{a} \lambda^{\eta^{(a)}}$. If this the case, and if we have an asymptotically free system for $f_{a}(\lambda) \equiv 0$, then also the corrected system is asymptotically free. Since the coefficients $C_{a}$ in the leading terms of $f_{a}(\lambda)$ for $\lambda \rightarrow 0$ is arbitrary, we have $n^{\prime}$ undetermined constants in the reduced solution, which must be fixed by information about the small couplings in order to get definite values for the corrections to the undisturbed reductions.

\section{EXAMPLES}

As we have mentioned in the introduction, there are many and varied applications of the reduction method. Here we can describe only two examples rather briefly.

Consider an $S U(2)$ gauge theory with one Dirac field and two scalar fields, all in the adjoint representation. The interaction part of the renormalizable theory is then given by

$$
\begin{aligned}
\mathcal{L}_{\text {int }} & =\text { gauge couplings }-i \sqrt{\lambda_{1}} \epsilon^{a b c} \bar{\psi}^{a}\left(A^{b}+i \gamma_{5} B^{b}\right) \psi^{c} \\
& -\frac{1}{4} \lambda_{2}\left(A^{a} A^{a}+B^{a} B^{a}\right)^{2}+\frac{1}{4} \lambda_{3}\left(A^{a} A^{b}+B^{a} B^{b}\right)^{2} .
\end{aligned}
$$

The algebraic reduction equations (14) have four solutions. With $\lambda=g^{2}$ as the primary coupling, $g$ being the gauge coupling, these solutions are power series in $g^{2}$ for $g^{2} \rightarrow 0$. After reparametrization, they are of the form [四]

$$
\text { (a) } \lambda_{1}=\lambda_{2}=\lambda_{3}=g^{2} \text {, }
$$

which corresponds to an $N=2$ extended SUSY Yang-Mills theory, and

$$
\text { (b) } \quad \lambda_{1}=g^{2}, \quad \lambda_{2}=\frac{9}{\sqrt{105}} g^{2}, \quad \lambda_{3}=\frac{7}{\sqrt{105}} g^{2}
$$


which is a new theory with one coupling and no supersymmetry. The other two solutions are obtained from (a) and (b) by reversing the sign of the quartic couplings $\lambda_{2}$ and $\lambda_{3}$, so that the classical potential approaches $-\infty$ with increasing scalar fields in almost all directions. Hence (a) and (b) are the only acceptable special solutions, which are power series in $g^{2}$ with real coefficients. Note that the Yukawa coupling $\left(\lambda_{1}\right)$ is required for the consistency of the reduction. There are no real solutions of the reduction equations for $\lambda_{1}=0$. The two reduced theories (31) and (32) can be considered as minimally coupled gauge theories with matter fields. As the original multiparameter theory, they are asymptotically free.

For the two solutions (31) and (32), the eigenvalues of the $3 \times 3$ matrix $\beta_{g 0}^{-1} M\left(f^{0}\right)$ are given by $\left(-2,-3, \quad+\frac{1}{2}\right)$ for $(\mathrm{a})$, and $\left(-2,-\frac{3}{4}(25 \pm\right.$ $\sqrt{345}) / \sqrt{105}$ ) for $(\mathrm{b})$. There are no positive integer eigenvalues. The only positive non-integer eigenvalue is $\eta=+\frac{1}{2}$ in (a). It gives rise to a general solution involving $\sqrt{g^{2}}$, which has one arbitrary parameter. This solution corresponds to a theory with hard SUSY breaking, which is consistent with the renormalization group.

Finally, I mention briefly the reduction [7] of the SU(5) minimal SUSY GUT [12]. The method of calculations is essentially along the lines we have discussed in Section 4. The reduction relates Yukawa couplings and the $\mathrm{SU}(5)$ gauge coupling above the unification scale. It is required to result in an asymptotically free theory. The results are used as boundary conditions for the conventional application of the renormalization group for scaling down to the Z-mass. Of course, a SUSY breaking scale enters into the calculation, but the results are not very sensitive to it.

I discuss here the gauge-Yukawa unification in the third generation as an example of the general reduction method. I do not consider the phenomenol- 
ogy in any detail, nor other approaches to the problem.

The original theory contains three Fermion families $(\overline{\mathbf{5}}+\mathbf{1 0})$, two Higgs supermultiplets $(\mathbf{5}+\overline{\mathbf{5}})$ for electroweak symmetry breaking, and a $\mathbf{2 4}$ multiplet for the spontaneous breaking $S U(5) \rightarrow S U(3) \times S U(2) \times U(1)$. Dimensionfull parameters and family mixing are neglected. There are six Yukawa couplings and two Higgs couplings. With SU(5) indices suppressed, the superpotential is exactly given by

$$
\begin{aligned}
W=\frac{1}{2}\left(g_{u} \mathbf{1 0}_{1} \mathbf{1 0}_{1}+\right. & \left.g_{c} \mathbf{1 0}_{2} \mathbf{1 0}_{2}+g_{t} \mathbf{1 0}_{3} \mathbf{1 0}_{3}\right) H \\
+\left(g_{d} \overline{\mathbf{5}}_{1} \mathbf{1 0}_{1}\right. & \left.+g_{s} \overline{\mathbf{5}}_{2} \mathbf{1 0}_{2}+g_{b} \overline{\mathbf{5}}_{3} \mathbf{1 0} \mathbf{0}_{3}\right) \bar{H} \\
& +\frac{g_{l}}{3}(\mathbf{2 4})^{3}+g_{f} \bar{H} \mathbf{2 4} H
\end{aligned}
$$

with $H$ and $\bar{H}$ denoting the $\mathbf{5}, \overline{\mathbf{5}}$ Higgs superfields. Using the one-loop $\beta$ functions for this minimal $\mathrm{SU}(5)$ model, we obtain the reduction equations

$$
\begin{aligned}
& \lambda \frac{d f_{u}}{d \lambda}=\frac{27}{5} f_{u}-3 f_{u}^{2}-\frac{4}{3} f_{u} f_{d}-\frac{8}{5} f_{u} f_{f} \\
& \lambda \frac{d f_{d}}{d \lambda}=\frac{27}{5} f_{d}-\frac{10}{3} f_{d}^{2}-f_{d} f_{u}-\frac{8}{5} f_{d} f_{f}
\end{aligned}
$$

and corresponding pairs of equations with $f_{u}, f_{d}$ replaced by $f_{c}, f_{s}$ or $f_{t}, f_{b}$ respectively. The notation is: $\lambda=g^{2} / 4 \pi ; \quad \lambda_{i}=\lambda f_{i}(\lambda), \quad i=u, d, c, s, t, b, l, f$. The remaining differential equations are

$$
\begin{aligned}
\lambda \frac{d f_{l}}{d \lambda} & =g f_{l}-\frac{21}{5} f_{l}^{2}-f_{l} f_{f}, \\
\lambda \frac{d f_{f}}{d \lambda} & =\frac{83}{15} f_{f}-\frac{53}{15} f_{f}^{2}-f_{f} f_{u}-\frac{4}{3} f_{f} f_{d}-\frac{7}{5} f_{f} f_{l}
\end{aligned}
$$

and corresponding equations involving $f_{c}, f_{s}$ and $f_{t}, f_{b}$.

The reduction is required to be compatible with the mass spectrum of the first two generations. This implies that the Yukawa couplings of these generations should be treated as small perturbations, so that the gauge Yukawa reduction is performed only for the third generation. Accordingly, 
we require

$$
\begin{array}{r}
f_{u}^{0}=f_{d}^{0}=f_{c}^{0}=f_{s}^{0}=0, \\
f_{t}^{0}>0, \quad f_{b}^{0}>0,
\end{array}
$$

and positive eigenvalues $\eta_{i}$ of the determinant, so that the corrections preserve the asymptotic freedom of the reduced theory. As we have seen in Section 4 , the functions $f_{a}(\lambda)$ with $f_{a}^{0}=0$ are of the form $f_{a}(\lambda)=C_{a} \lambda^{\eta_{a}}+\cdots$.

With the conditions described above, the a priori large number of solution of the equations (34,35) is reduced to two, which are given by $\lambda_{\alpha}=\lambda f_{\alpha}^{0}, \quad \alpha=$ $t, b, l, f$, with

$$
\begin{aligned}
& \text { (1) } f_{t}^{0}=\frac{2533}{2605}=0.97, \quad f_{b}^{0}=\frac{1491}{2605}=0.57, \quad f_{l}^{0}=0, \quad f_{f}^{0}=\frac{560}{521} \\
& \text { (2) } f_{t}^{0}=\frac{89}{65}=1.37, \quad f_{b}^{0}=\frac{63}{65}=0.97, \quad f_{l}^{0}=0, \quad f_{f}^{0}=0
\end{aligned}
$$

The numbers for the positive eigenvalues $\eta_{i}$ are given in [0]]

We can now use the solutions (37) in order to obtain reduction formulas including small corrections. The method has been described in Section 4 . As shown in detail in [7], on the basis of one-loop information, we find that the partial differential equations (28) have unique power series solutions of the form (29), with coefficients which are again power series in the small couplings. It turns out that the contributions from the Yukawa couplings of the first two generations are negligible. Hence we obtain the solutions

$$
\begin{aligned}
\text { (1) } f_{\alpha} & =f_{\alpha}^{0}+\chi_{l \alpha}^{(1)} f_{l}+\chi_{l \alpha}^{(2)} f_{l}^{2}+\cdots, \\
\alpha & =t, b, f \\
(2) f_{\alpha} & =f_{\alpha}^{0}+\chi_{l \alpha}^{(1)} f_{l}+\chi_{l f \alpha}^{(11)} f_{l} f_{f}+\chi_{l \alpha}^{(2)} f_{l}^{2}+\chi_{f \alpha}^{(2)} f_{f}^{2}+\cdots, \\
\alpha & =t, b .
\end{aligned}
$$

The coefficients $\chi$ are determined, and we have $f_{l}=C_{l} \lambda^{\eta_{l}}, \quad f_{f}=C_{f} \lambda^{\eta_{f}}$ with positive eigenvalues $\eta$, while $C_{l}$ and $C_{f}$ are to be fixed by input data. 
Actually, the two special solutions listed above are boundaries of the same, asymptotically free general solution.

The solutions (37) for the reduction with corrections of the minimal SU(5) model are valid at the unification scale $\left(\sim 10^{25} \mathrm{GeV}\right)$. As mentioned before, they can be used as input for the usual renormalization group equations of the minimal supersymmetric standard model, which are set up to scale the reduced theory down to energies of the order of $M_{Z}$. A SUSY breaking mechanism must be introduced, so that the superpartners are unobservable at lower energies. There are other requirements, like the suppression of proton decay, which must be taken into account. With the input data

$$
\begin{gathered}
\sin ^{2} \theta_{W}\left(M_{Z}\right)=0.2315, \alpha_{\mathrm{em}}^{-1}\left(M_{Z}\right)=128.09 \\
m_{\tau}=1.777 \mathrm{GeV}, M_{Z}=91.188 \mathrm{GeV},
\end{gathered}
$$

and SUSY breaking scales of 300 or $500 \mathrm{GeV}$, the main results obtained by Kubo, Mondragón and Zupanos [ pre

(1) $m_{t}=179.0 \mathrm{GeV}, m_{b}=5.5 \mathrm{GeV}, \alpha_{S}\left(M_{Z}\right)=0.123$

(2) $m_{t}=182.3 \mathrm{GeV}, m_{b}=5.4 \mathrm{GeV}, \alpha_{S}\left(M_{Z}\right)=0.123$

for $M_{\mathrm{SUSY}}=300 \mathrm{GeV}$, and

$$
\begin{aligned}
& \text { (1) } m_{t}=179.0 \mathrm{GeV}, m_{b}=5.5 \mathrm{GeV}, \alpha_{S}\left(M_{Z}\right)=0.122 \\
& \text { (2) } m_{t}=182.6 \mathrm{GeV}, m_{b}=5.3 \mathrm{GeV}, \alpha_{S}\left(M_{Z}\right)=0.122
\end{aligned}
$$

for $M_{\mathrm{SUSY}}=500 \mathrm{GeV}$.

\footnotetext{
${ }^{3}$ I am very grateful to Jisuke Kubo for kindly providing me with the data from the latest recalculation. These results are essentially the same as those obtained in March 1994 [13, Ø].
} 
Here the masses are the pole masses, and $\alpha_{S}\left(M_{Z}\right)$ is defined in the $\overline{\mathrm{MS}}$ scheme with five flavors. These results are obtained in an asymptotically free, renormalizable, reduced theory. Reduction calculation have also been performed starting from different initial models [14]. The results are quite similar.

As I have mentioned before, this talk is mainly concerned with a description of the reduction method, and not with phenomenology. But in order to assess the results for the top-bottom hierarchy, it is relevant to consider an aspect of the renormalization group equations for scaling the Yukawa couplings from the GUT scale to the scale of the $Z$-mass. This scaling is done within the framework of the minimal supersymmetric standard model. It turns out that, for sufficiently large values of the top-Yukawa coupling $\lambda_{t}$ at the unification scale, the results obtained for $m_{t}\left(M_{Z}\right)$ are rather insensitive to the precise value of this coupling. Consequently, calculations of the coupling $\lambda_{t}$ can be tested more sensitively if the experimental top-quark mass is at least slightly smaller than the value corresponding to the plateau of the curve $m_{t}\left(M_{Z}\right)$ versus $\lambda_{t}$. 自

The reduction method has previously been applied to the standard theory for electroweak and strong interactions [8]. Extensive calculations, including all corrections, give top masses (about $100 \mathrm{GeV}$ ) well below the experimental value. This is an important result, because it indicates that more fields are needed, which are provided for in the SUSY models described above.

\footnotetext{
${ }^{4}$ In the literature, this value of the top-quark mass is usually called the "quasi infrared fixed point" solution [15].
} 


\section{ACKNOWLEDGMENTS}

For conversations and remarks. I am indebted to K. Sibold and W. Zimmermann. I am particularly grateful to Jisuke Kubo for many helpful communications. It is a pleasure to thank Wolfhart Zimmermann, and the theory group of the Max Planck Institut für Physik, Werner Heisenberg Institut, for their kind hospitality in München.

This work has been supported in part by the National Science Foundation, grant PHY 91-23780. 


\section{References}

[1] R. Oehme and W. Zimmermann, Max Planck Institut Report MPTPAE/Pth 60/82 (1982), Commun. Math. Phys. 97 (1985) 569; R. Oehme, K. Sibold and W. Zimmermann, Phys. Lett. B147 (1984) 115; W. Zimmermann, Commun. Math. Phys. 97 (1985) 211; R. Oehme, K. Sibold and W. Zimmermann, Phys. Lett. B153 (1985) 142; R. Oehme, Prog. Theor. Phys. Suppl. 86 (1986) 215.

[2] N.-P. Chang, Phys. Rev. D10 (1974) 2706; N.-P. Chang, A. Das and J. Perez-Mercader, Phys. Rev. D22 (1980) 1829.

[3] R. Oehme, "Reduction in Coupling Parameter Space", in Anomalies, Geometry and Topology, proceedings, ed. A. White (World Scientific, Singapore, 1985) pp. 443-458;

W. Zimmermann, "Renormalization Group and Symmetries in Quantum Field Theory", in Proceedings of the 14th ICGTMP, ed. Y. M. Cho (World Scientific, Singapore, 1985) pp. 145-154;

K. Sibold, "Reduction of Couplings", Acta Physica Polonica, 19 (1988) 295.

[4] R. Oehme, K. Sibold and W. Zimmermann, Phys. Lett. B153 (1985) 142.

[5] C. Lucchesi, O. Piguet and K. Sibold, Phys. Lett. B201 (1988) 241;

Helv. Physica Acta 61 (1988) 321;

C. Lucchesi, "All Orders Finite N = 1 Super-Yang-Mills Theories", SUSY 95, Paris; NEIP-95-012, hep-th/9510078. 
[6] K. G. Wilson, T. S. Walhout, A. Harianas, W-M. Zhang and R. J. Perry, Phys. Rev. D49 (1994) 6720; R. J. Perry and K. G. Wilson, Nucl. Phys. B403 (1993) 587; R. J. Perry, Ann. of Physics (N.Y.), 232 (1994) 116.

[7] J. Kubo, M. Mondragón and G. Zupanos, Nucl. Phys. B424 (1994) 291; "Finite Unification and Top Quark Mass", in Proceedings of the 27th Int. Conf. on High Energy Physics (Glasgow, 1994).

[8] J. Kubo, K. Sibold and W. Zimmermann, Nucl. Phys. B259 (1985) 331; Phys. Lett. B220 (1989) 185; K. Sibold and W. Zimmermann, Phys. Lett. B191 (1987) 427; J. Kubo, Phys. Lett. B262 (1991) 472; W. Zimmermann, Phys. Lett. B311 (1993) 249.

[9] R. Oehme, Prog. Theor. Phys. Suppl. 86 (1986) 215.

[10] I. Jack and D.R.T. Jones, Phys. Lett. B333 (1994) 372 ; Phys. Lett. B349 (1995) 294.

[11] W. Zimmermann, Phys. Lett. B311 (1993) 249.

[12] S. Dimopoulos and H. Georgi, Nucl. Phys. B193 (1981) 150 ; N. Sakai, Z. Phys. C11 (1981) 153 .

[13] J. Kubo, M. Montragón and G. Zupanos, Max Planck Institut Report MPI-Ph/94-10, March 1994.

[14] J. Kubo, M. Mondragón and G. Zupanos, "Gauge-Yukawa Unification", in Proceedings of SUSY 95 ( Ecole Polytechnique, Palaiseau. France, May 1995).

[15] C.T. Hill, Phys. Rev. D24 (1981) 691; C.T. Hill, C.N. Leung and S. Rao, Nucl. Phys. B262 (1985) 517; W.A. Bardeen, M. Carena, S. Pokorsky 
and C.E.M. Wagner, Phys. Lett. B320 (1994) 110.

J. Kubo, M. Mondragón, M. Olechowsky and G. Zoupanos, "GaugeYukawa Unification and the Top-Bottom Hierarchy" in Proceedings of the International Europhysics Conference on HEP (Brussels, JulyAugust 1995).

These papers contain further references. 